\title{
Análise da saúde dos profissionais de enfermagem no contexto da pandemia SARS- CoV-2
}

\author{
Analysis of the health of nursing professionals in the context of the SARS-CoV-2 \\ Análisis de la salud de los profesionales de enfermería en el contexto de la pandemia SARS-CoV-2
}

Recebido: 03/11/2021 | Revisado: 09/11/2021 | Aceito: 17/11/2021 | Publicado: 19/11/2021

Joelma de Carvalho Neres

ORCID: https://orcid.org/0000-0003-4317-0600

Universidade Paulista, Brasília

E-mail: Joelmacarvalhoneres99@gmail.com

Larissa dos Santos Lopes

ORCID: https://orcid.org/0000-0002-5772-4128

Universidade Paulista, Brasília

E-mail: larissalopesmg25@gmail.com

Maria Luiza Rêgo Bezerra

ORCID: https://orcid.org/0000-0002-3336-7760 Universidade Paulista, Brasília

E-mail: maria.bezerra@docente.unip.br

Maryane Fernanda Pereira de Lima

ORCID: https://orcid.org/0000-0002-1952-7924 Universidade Paulista, Brasil

E-mail: Fernanda-maryane@ @otmail.com

Tássila Evelyn Alves de Oliveira

ORCID: https://orcid.org/0000-0002-7218-7076 Universidade Paulista, Brasil

E-mail: tassila.evelyn.alves@gmail.com

\begin{abstract}
Resumo
Objetivo: Descrever os impactos causados na saúde mental dos profissionais de enfermagem na pandemia da Covid19. Método: Trata-se de um estudo de revisão integrativa de literatura, voltada aos impactos que a pandemia trouxe à saúde mental dos enfermeiros. Foi realizado um levantamento de artigos publicados na base de dados da Literatura Latino-Americana e do Caribe em Ciência da Saúde, Biblioteca Eletrônica Cientifica Online, Bases de Dados de Enfermagem e Medical Literature Analysis and Retrievel System Online. Resultados: Foram incluídos em 15 publicações na amostra final do estudo. Identificação das causas e dificuldades como: exposição dos profissionais ao vírus, longas jornadas de trabalho, pouca quantidade de recursos, sofrimentos dos pacientes, preocupação em contaminar os familiares, pressão psicológica pela grande responsabilidade em salvar vidas. Conclusão: A pandemia pelo COVID-19, a exposição dos profissionais de enfermagem as condições excessivas de trabalho, o contato direto com pacientes infectados, trouxeram situações como adoecimento e quantidade insuficiente de profissionais para atender a demanda. $\mathrm{O}$ apoio psicológico para esses profissionais e melhores condições de trabalho são de suma importância nesse contexto de pandemia.
\end{abstract}

Palavras-chave: Saúde mental; Profissional de enfermagem; Covid-19.

\begin{abstract}
Objective: To describe the impacts caused on the mental health of nursing professionals in the Covid-19 pandemic. Method: This is an integrative literature review study, focused on the impacts that the pandemic brought to the mental health of nurses. A survey of articles published in the database of Latin American and Caribbean Literature in Health Science, Scientific Electronic Library Online, Nursing Databases and Medical Literature Analysis and Retrievel System Online was carried out. Results: 15 publications were included in the final study sample. Identification of causes and difficulties such as: exposure of professionals to the virus, long working hours, limited resources, patients' suffering, concern with contaminating family members, psychological pressure for the great responsibility to save lives. Conclusion: The COVID-19 pandemic, the exposure of nursing professionals to excessive working conditions, direct contact with infected patients, brought situations such as illness and insufficient number of professionals to meet the demand. Psychological support for these professionals and better working conditions are of paramount importance in this pandemic context.
\end{abstract}

Keywords: Mental health; Nursing professional; Covid-19.

\section{Resumen}

Objetivo: Describir los impactos causados en la salud mental de los profesionales de enfermería en la pandemia Covid19. Método: Se trata de un estudio de revisión integradora de la literatura, centrado en los impactos que la pandemia 
trajo a la salud mental de las enfermeras. Se realizó una encuesta de artículos publicados en la base de datos de Literatura Latinoamericana y Caribeña en Ciencias de la Salud, Biblioteca Electrónica Científica en Línea, Bases de Datos de Enfermería y Medical Literature Analysis and Retrievel System Online. Resultados: se incluyeron 15 publicaciones en la muestra final del estudio. Identificación de causas y dificultades como: exposición de los profesionales al virus, largas jornadas laborales, recursos limitados, sufrimiento de los pacientes, preocupación por contaminar familiares, presión psicológica por la gran responsabilidad de salvar vidas. Conclusión: La pandemia de COVID-19, la exposición de los profesionales de enfermería a condiciones laborales excesivas, el contacto directo con pacientes infectados, trajo situaciones como enfermedad y número insuficiente de profesionales para atender la demanda. El apoyo psicológico a estos profesionales y la mejora de las condiciones laborales son de suma importancia en este contexto pandémico.

Palabras clave: Salud mental; Profesional de enfermería; Covid-19.

\section{Introdução}

Em dezembro de 2019, foi localizado em Wuhan na China, um surto de uma nova doença, provocada pelo vírus SARSCOV-2 (anteriormente conhecido como 19-NCOV). Em janeiro de 2020 foi confirmada a existência de uma epidemia, provocada pelo vírus e em março de 2020 a doença tomou maiores proporções sendo disseminada para centenas de países, tornando-se uma grande pandemia (Werneck \& Carvalho, 2020). Devido ao insuficiente conhecimento científico sobre a Covid-19, a sua alta velocidade de disseminação e a gravidade da doença, foram geradas grandes incertezas sobre a estratégia a ser utilizada no enfrentamento da doença em todo o mundo. Diante do cenário atual, devido ao enfrentamento de longas jornadas de trabalho, poucos recursos, uso de EPI desconfortável, sofrimento das vítimas e preocupação com a possibilidade de infectar os familiares, os profissionais da saúde carregam uma grande carga de estresse (Werneck \& Carvalho, 2020; Ornell, Halpern, Kessler, \& Narvaez, 2020).

O estresse é gerado por uma série de estímulos externos que desequilibram o estado emocional de um indivíduo. Esse estado emocional desequilibrado resulta em inúmeros distúrbios fisiológicos e psicológicos, e caso seja relacionado ao trabalho é denominado estresse ocupacional. Segundo a OMS (Organização Mundial de Saúde) cobrança e pressão excessivas resultam em manifestação do estresse, podendo vir a causar o desequilíbrio emocional do profissional (Mota et. al., 2021). São muitos os fatores que contribuem para a formação do estresse na enfermagem. Muitos estão relacionados com a própria profissão, como por exemplo, lidar com a dor e sofrimento, com pacientes terminais, e a sobrecarga de trabalho acabam trazendo como consequência diversos transtornos psicológicos relacionados ao estresse. Alvares, Thomaz, Lamy, Nina, \& Pereira, 2020).

O efeito do estresse ocupacional causados aos profissionais da enfermagem vem sendo muito estudado nos dias de hoje, por ser um importante problema de saúde principalmente no cenário de pandemia que se vive atualmente. O estresse ocupacional se dá quando há percepção do profissional de que o local de trabalho ameaça sua saúde mental, pela demanda excessiva de trabalho ou por outros motivos. (Alvares, Thomaz, Lamy, Nina, \& Pereira, 2020). A contingência hospitalar aponta a rápida incidência de casos e superlotação nos serviços de saúde pública, principalmente hospitalares e em especial unidades de terapias intensivas. Sendo apontado um grande desafio no setor público pela escassez principalmente de leitos suprimentos (Santos, et. al., 2021).

Dessa forma, elaborou-se a seguinte pergunta-problema: Quais as evidências encontradas na literatura científica, nacional e internacional, acerca da repercussão na análise da saúde mental dos profissionais de saúde na pandemia SARS-COV19 e medidas a serem adotadas para evitar o estresse ocupacional nos profissionais de enfermagem?

A presente pesquisa tem como finalidade trazer uma amplitude maior de conhecimento, abordando a importância da adoção de medidas por parte das organizações laborais, como por exemplo, maior capacitação dos trabalhadores de forma que seja reduzida a transmissão da doença, pois o estresse pode levar à consequências muito graves e em casos mais extremos o suicídio. O estudo se faz relevante para identificar a partir da uma revisão integrativa de literatura o impacto da pandemia de convid-19 na saúde mental dos profissionais de enfermagem. 


\section{Metodologia}

Trata-se de uma revisão integrativa da literatura no que se refere à partir da Análise da saúde mental dos profissionais de enfermagem no contexto da pandemia SARS-COV-19. Tem como finalidade reunir e sintetizar estudos anteriores contribuindo para conhecimento sobre o tema escolhido através de uma revisão integrativa da literatura, a produção de saberes acerca da repercussão. Iniciou-se a operacionalização da pesquisa, através da busca de Descritores em Ciências da Saúde (DeCS), por meio da Biblioteca Virtual em Saúde (BVS), utilizando bancos de dados como Literatura Latino-Americana e do Caribe em Ciências da Saúde (LILACS), Scientific Electronic Library Online (Scielo), Base de Dados de Enfermagem (BDENF), Medical Literature Analysis and Retrieval System Online (MEDLINE), utilizando as seguintes e palavras chaves: Saúde Menta; Profissional de Enfermagem; Covid-19.

Os critérios de inclusão foram: artigos publicados na íntegra e disponibilizados pública e gratuitamente, em inglês, português e espanhol, e cujos resultados ou reflexões pautam sobre análise da saúde mental dos profissionais de enfermagem na pandemia da COVID-19, foram excluídas reportagens e demais materiais informativos, governamentais, artigos duplicados e midiáticos. Na coleta de dados, realizou-se a busca ampla nas bases de dados, a partir dos descritores "and saúde mental, and profissional de enfermagem, and covid-19", sem critérios de refinamento, encontrados um total de 156 artigos.

Os estudos que preencheram os critérios de inclusão e cruzamento dos descritores foram artigos publicados nos períodos de 2020 e 2021. Chegou-se a um artigos da SciELO, quinze artigos da BDENF-Enfermagem, dezesseis artigos da LILACS, e cento e vinte e oito MEDLINE. Totalizando 160 artigos. Desta forma, 15 artigos totalizaram a amostra final desta revisão. A partir da adequação da busca à questão norteadora deste estudo chegou-se a 145 artigos, sendo 130 repetidos nas diferentes bases de dados. Para melhor visualização das etapas de seleção e análise dos dados, utilizou-se o fluxograma Prisma Statement, conforme Figura 01.

Figura 1. Fluxograma coleta e análise dos dados - Brasília-DF - 2021.

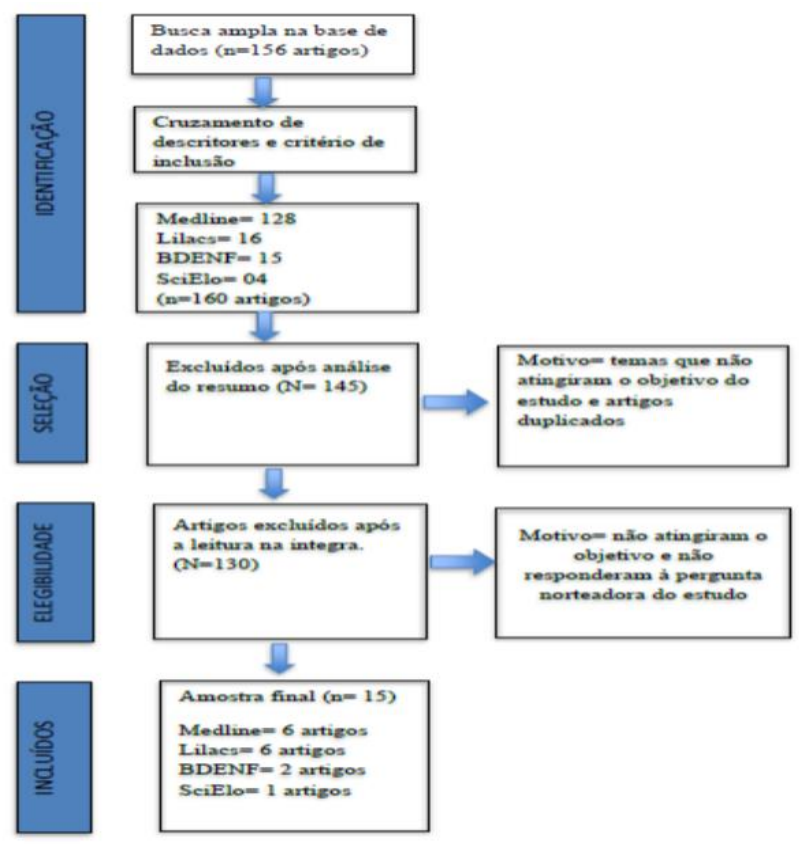

Fonte: Autoras (2021).

\section{Resultados e Discussão}

Apresentaremos o Quadro 1 com os dados dos 15 artigos selecionados, sendo esses referentes a SAUDE MENTAL, 
ENFERMAGEM e COVID-19, descrevendo assim as características dos estudos elegíveis descritos abaixo.

Quadro 1 - Características dos estudos selecionados- Brasília-DF - 2021.

\begin{tabular}{|c|c|c|c|c|}
\hline Título & $\begin{array}{l}\text { Periódico e ano de } \\
\text { Publicação }\end{array}$ & Origem & Abordagem & Contexto \\
\hline $\begin{array}{l}\text { Trabalho da enfermagem na pandemia da covid-19 e repercussão } \\
\text { para a saúde mental dos trabalhadores. }\end{array}$ & $\begin{array}{l}2021 \text { Rev. Gaúcha de } \\
\text { enfermagem }\end{array}$ & Brasil & Qualitativo & Cuidado \\
\hline $\begin{array}{l}\text { Nurses perspectives of taking care of patients with Coronavirus } \\
\text { disease 2019: A phenomenological study. }\end{array}$ & 2021 Rev. PLOS ONE & EUA & Qualitativo & Cuidado \\
\hline $\begin{array}{l}\text { Experiences of renal healthcare practitioners during the COVID- } \\
19 \text { pandemic: a multi-methods approach. }\end{array}$ & 2021 BMC Nephrology & EUA & Misto & Cuidado \\
\hline $\begin{array}{l}\text { Practical strategies and the need for psychological support: } \\
\text { recommendations from nurses working in hospitals during the } \\
\text { COVID-19 pandemic. }\end{array}$ & $\begin{array}{l}2021 \text { Journal of Health } \\
\text { Organization and } \\
\text { Management }\end{array}$ & EUA & Qualitativo & Cuidado \\
\hline $\begin{array}{l}\text { A qualitative exploration of the experiences of school nurses } \\
\text { during COVID-19 pandemic as the frontline primary health care } \\
\text { professionals. }\end{array}$ & $\begin{array}{l}2021 \text { Rev. Nursing } \\
\text { outlook }\end{array}$ & EUA & Qualitativo & Cuidados \\
\hline $\begin{array}{l}\text { Nurses' barriers to caring for patients with COVID-19: a } \\
\text { qualitative systematic review. }\end{array}$ & $\begin{array}{l}2021 \text { Wiley online } \\
\text { library }\end{array}$ & EUA & Misto & Cuidado \\
\hline Burnout e saúde mental em tempos de pandemia de COVID - 19. & 2021 Revista Nursing & Brasil & Qualitativo & Cuidado \\
\hline $\begin{array}{l}\text { Sofrimento psíquico entre os profissionais de enfermagem } \\
\text { durante a pandemia da COVID- } 19 \text {. }\end{array}$ & 2021 Escola Anna Neri & Brasil & Misto & Cuidado \\
\hline Atuação da enfermagem no cenário da pandemia COVID-19. & 2020 Revista Nursing & Brasil & Qualitativo & Cuidado \\
\hline $\begin{array}{l}\text { Condições de trabalho e o impacto na saúde dos profissionais de } \\
\text { enfermagem frente a covid- } 19 \text {. }\end{array}$ & $\begin{array}{l}2020 \text { Cogitare } \\
\text { Enfermagem }\end{array}$ & Brasil & Quantitativo & Cuidado \\
\hline $\begin{array}{l}\text { Hospital-Based Health Care Worker Perceptions of Personal } \\
\text { Risk Related to Covid-19. }\end{array}$ & $\begin{array}{c}2020 \text { Internal medicine } \\
\text { journal }\end{array}$ & EUA & Qualitativo & Cuidado \\
\hline $\begin{array}{l}\text { Escuta empática: Estratégia de acolhimento aos profissionais de } \\
\text { enfermagem no enfrentamento da pandemia por coronavírus. }\end{array}$ & $\begin{array}{l}2021 \text { Rev. Brasileira de } \\
\text { Enfermagem }\end{array}$ & Brasil & Qualitativo & Cuidado \\
\hline $\begin{array}{l}\text { Cuidados de enfermagem prestados a pessoas com covid- } \\
\text { 19:Desafios no desempenho do sistema COFEN/CORENS }\end{array}$ & $\begin{array}{l}2021 \text { Rev. Texto \& } \\
\text { Contexto - } \\
\text { Enfermagem }\end{array}$ & Brasil & Qualitativo & Cuidado \\
\hline Nurses' Mental Health During the Covid-19 Outbreak & $\begin{array}{l}2020 \text { Journal of } \\
\text { Occupational and } \\
\text { Environmental } \\
\text { Medicine }\end{array}$ & EUA & Quantitativo & Cuidado \\
\hline $\begin{array}{l}\text { O 'NOVO' da COVID-19: impactos na saúde mental de } \\
\text { profissionais de enfermagem? }\end{array}$ & $\begin{array}{l}2021 \text { Acta Paulista de } \\
\text { Enfermagem }\end{array}$ & Brasil & Qualitativo & Cuidado \\
\hline
\end{tabular}

Fonte: Autoras (2021).

Esta revisão tem como objetivo, trazer uma discussão sobre as evidências encontradas acerca da repercussão na análise da saúde mental dos profissionais de saúde na pandemia SARS-COV-19 e medidas a serem adotadas para evitar o estresse. Sabese que a doença SARS-COV-2 é transmitida através de gotículas e aerossóis. Dessa forma, os profissionais de enfermagem que são linha de frente em cuidado, acabam sendo os profissionais mais contaminados, sendo necessário uma maior proteção com os EPI's adequados que seriam: máscara, capotes de manga longa, luvas e óculos para proteção do trabalhador (Souza, et. al., 2021).

As organizações laborais devem adotar medidas para melhor capacitação dos trabalhadores a fim de reduzir a transmissão e contágio da doença (Rathnayake, Dasanayake, Maithreepala, Ekanayake, \& Basnayake, 2021). Durante as pesquisas notou-se que durante a pandemia houve um maior índice de problemas psicológicos entre os enfermeiros, pois os mesmos relatam medo agravado por conta do risco de contaminação dos familiares. Além disso, relatou-se sentimento de culpa em relação ao cuidado com o paciente (Mc, et al., 2021)

Segundo os profissionais entrevistados, falta uma melhor comunicação das políticas hospitalares onde são relacionadas às licenças médicas, remuneração e carga de trabalho. Esses mesmos profissionais também relatam a necessidade de apoio psicológico que deveria ser oferecido pelos provedores (Ralph, Freeman, Ménard, \& Soucie, 2021). A grande maioria dos 
participantes relatam sentimento de ansiedade, estresse, incerteza, mal-estar físico, sobrecarga de informações pelo fato de ser um vírus novo e as informações muito confusas sobre a transmissão do vírus (Lee, et. al., 2021).

No Brasil, é inexistente os dados que referem-se ao adoecimento dos profissionais de enfermagem, porém o Conselho Federal de Enfermagem confirmou 143 mortes de profissionais e 17.044 casos de infectados. Registram-se elevado número de profissionais com problemas psicológicos como a Síndrome de Burnout, depressão, Síndrome do pânico, entre outras que atingem a saúde mental (Joo \& Liu, 2021). Em todos os estudos relacionados a essa temática notou-se a falta de apoio dos hospitais e do sistema de saúde aos enfermeiros. Os profissionais relatam a falta de equipamentos de proteção individual, falta de unidades de isolamento para acomodação dos pacientes com COVID-19, sobrecarga de trabalho pela falta de profissionais nas unidades (Joo \& Liu, 2021).

Normalmente a enfermagem é exposta a situações de grande estresse e diante da situação pandêmica foram acentuadas a exaustão emocional dos profissionais (Luz, et. al., 2021). Vale ressaltar que os profissionais tendem a deixar de cuidar de sua própria saúde mental devido a rotina cansativa (Miranda, Yamura, Pereira, Pereira, \& Zanatta 2021). A enfermagem enfrenta rotinas exaustivas fazendo com quem fiquem suscetíveis a desenvolver a problemas psicológicos causados pelo estresse. Diante desse contexto cabe mencionar que existem várias recomendações sobre a importância da saúde mental da equipe de enfermagem, uma vez que eles representam a linha de frente de qualquer serviço de saúde. Assim, é preciso que sejam implementadas estratégias de apoio a essa categoria, e essas estratégias devem ser permanentes, o que seria necessário maior investimento das instituições públicas e privadas (Reis, Lago, Carvalho, Nobre, \& Guimarães, 2020)

Apesar das adversidades, a enfermagem vem sendo protagonista no enfrentamento da pandemia nas ações de vigilância, prevenção e controle da transmissão do vírus (Miranda, Santana, Pizzolato, \& Saquis, 2020).

Nessa crise pandêmica é preciso cuidar da saúde mental de quem cuida, sempre fortalecendo o trabalho em equipe para uma melhor promoção de saúde dos enfermeiros (Chu, et. al., 2021).

Este estudo explicitou-se a precariedade que há décadas o sistema de saúde brasileiro vem sofrendo o que com a pandemia da COVID-19 acabou piorando. Isso acabou se tornando um dos fatores determinantes para o sofrimento psíquico dos trabalhadores de enfermagem (Tobase, Rodrigues, \& Peres, 2021).

$\mathrm{m}$ todos os achados nas pesquisas foram confirmados os impactos na saúde mental dos profissionais de enfermagem no contexto da COVID-19. Consta-se que 65,6\% dos profissionais que atuam na assistência direta a mais de 14 anos são os mais afetados (Clementino, et. al., 2020).

Os problemas mais encontrados entre os enfermeiros durante as pesquisas são: - O surto da COVID-19 trouxe altos níveis de ansiedade, depressão e estresse; - Más condições de trabalho, horas extras levantaram a níveis elevados de depressão e ansiedade; - Falta de EPI adequado contribuem para o estresse (Sampaio, Sequeira \& Teixeira, 2020; Queiroz, 2021).

Sugere -se para diminuição desses problemas que sejam desenvolvidas nossos métodos que recebem os reais impactos da pandemia nessa categoria profissional. A proteção dos profissionais de saúde é de suma importância, uma vez que são linha de frente para lidar com o surto desse vírus. Melhores condições de trabalho, salário digno, EPI'S adequados e valorização da classe são cruciais para proteger a saúde mental da enfermagem (Sampaio, Sequeira \& Teixeira, 2020; Queiroz, 2021).

\section{Conclusão}

Surge em 2019 um vírus capaz de mudar o percurso natural da história. Em um mercado chinês acontece a primeira notificação de pessoas infectadas, no ano seguinte cresce exponencialmente o número de casos saindo de uma epidemia local para uma pandemia mundial. Em detrimento dessas circunstâncias começam a surgir um número exorbitante de óbitos e superlotação nas redes hospitalares em todo o mundo. 
A pandemia pelo COVID 19, a exposição dos profissionais de enfermagem as condições excessivas de trabalho, o contato direto com pacientes infectados, trouxeram situações como adoecimento e quantidade insuficiente de profissionais para atender a demanda. Isso acabou dificultando a implementação de estratégias de segurança ao paciente quanto ao seu cuidado.

Sobretudo desencadeando grandes transtornos mentais, desenvolvendo problemas que acabam se tornando crônicos como burnot, depressão e ansiedade. Ao longo do presente estudo pôde ser evidenciado de forma significativa o sofrimento psicológico que a pandemia trouxe aos profissionais de enfermagem, durante o seu exercício profissional.

Salienta-se que grande parte desse sofrimento tem como causa a desvalorização profissional, a sobrecarga de trabalho e a pressão psicológica enfrentadas por esses profissionais.

Conclui-se, que para minimizar esses tipos de transtornos sugere-se aos profissionais de enfermagem a realização de mobilizações em prol da melhoria de condições de trabalho, EPI's adequados e um correto dimensionamento de profissionais.

Alertar as autoridades sobre a saúde mental da enfermagem reivindicando os fluxos de atendimento e melhoria nas condições de trabalho, também são de grande importância, uma vez que estes profissionais são de fundamental importância para a qualidade do cuidado.

\section{Referências}

Alvares, M. E. M., Thomaz, E. B. A. F., Lamy, Z. C., Nina, R. V. A. H, \& Pereira, M. U. L. (2020). Síndrome de burnout entre profissionais de saúde nas unidades de terapia intensiva: um estudo transversal com base populacional. Revista Brasileira de Terapia Intensiva, 32 (2): 251-260

Chu, E., Lee, K. M., Stotts, R., Benjenk, I., Ho, G., Yamane, D., Mullins, B., \& Heinz, E. R. (2021) Hospital-Based Health Care Worker Perceptions of Personal Risk Related to COVID-19. J Am Board Fam Med. S103-S112. 10.3122/jabfm.2021.S1.200343. PMID: 33622825

Clementino, F. S., Chaves, A. E. P., Pessoa Júnior J. M., Miranda, F. A. N, Medeiros, S. M., \& Martiniano C. S. (2020). Enfermagem na atenção às pessoas com COVID-19: desafios na atuação do sistema COFEN/CORENS. Texto Contexto Enferm, 29: e20200251. https://doi.org/10.1590/1980-265X-TCE-2020-0251

Joo, J. Y.\& Liu, M. F. (2021) Nurses' barriers to caring for patients with COVID-19: a qualitative systematic review. Int. Nurs.Rev, 68, 202-213

Lee, R. L. T., West, S., Tang, A. C. Y., Cheng, H. Y., Chong, C. Y. Y., Chien, W. T., \& Chan, S. W. C. (2021). A qualitative exploration of the experiences of school nurses during COVID-19 pandemic as the frontline primary health care professionals. Nurs Outlook, 69(3), 399-408. https://doi.org/ 10.1016/j.outlook.2020.12.003

Luz, D. C. R. P., Campos, J. R. E., Bezerra, P. O. S., Campos, J. B. R., Nascimento, A. M. V., \& Barros, A. B. (2021). Burnout e saúde mental em tempos de pandemia de COVID -19: revisão sistemática com metanális. Revista Nursing, 24 (276): 5714-571

Mc, C. K., Reid, J., Carswell, C. et al. (2021) Experiências de profissionais de saúde renal durante a pandemia de COVID-19: uma abordagem multimétodos. BMC Nephrol 22, 301. https://doi.org/10.1186/s12882-021-02500-0

Miranda, F. B. G., Yamura, M., Pereira, S. S., Pereira, C. S., Zanatta, S. T. P., \& Costa, M. K. \& Zerbetto, S. R. (2021). Sofrimento psíquico entre os profissionais de enfermagem durante a pandemia da COVID-19: Scoping Review. Escola Anna Nery, 25, e20200363. https://doi.org/10.1590/2177-9465-EAN-2020-0363

Miranda, F. M. A., Santana, L. M., Pizzolato, A. C., \& Saquis, L. M. M. (2020). Condições de trabalho e o impacto na saúde dos profissionais de enfermagem frente a Covid-19. Cogitare enferm, 25: e72702. http://dx.doi.org/10.5380/ce.v25i0.72702

Mota, R. S., Silva, V. A. da, Brito, I. G., Barros, A. de S., Santos, O. M. B. dos, Mendes, A. S., \& Souza, L. de C. (2021). estresse ocupacional relacionado à assistência de enfermagem em terapia intensiva. Revista Baiana De Enfermagem35 ,. https://doi.org/10.18471/rbe.v35.38860

Ornell, F., Halpern, S. C., Kessler, F. H. P., \& Narvaez J. C. M. (2020). O impacto da pandemia de COVID-19 na saúde mental dos profissionais de saúde. Cadernos de Saúde Pública, 36 (4): e00036520

Queiroz, A. M., Sousa, A. R., Moreira, W. C, Nóbrega, M. P., Santos, M. B., Barbossa, L. J., et al. (2021) O 'NOVO’ da COVID-19: impactos na saúde mental de profissionais de enfermagem? Acta Paul Enferm. ,34:eAPE02523

Ralph, J., Freeman, L. A., Ménard, A. D. \& Soucie, K. (2021), "Estratégias práticas e a necessidade de apoio psicológico: recomendações de enfermeiras que trabalham em hospitais durante a pandemia de COVID-19", Journal of Health Organization e Management. https://doi.org/10.1108/JHOM-02-2021-0051

Rathnayake, S., Dasanayake, D., Maithreepala, S. D., Ekanayake, R., \& Basnayake, P. L. (2021) Nurses' perspectives of taking care of patients with Coronavirus disease 2019: A phenomenological study. PLOS ONE, 16(9): e0257064. https://doi.org/10.1371/journal. pone.0257064

Reis, L. M., Lago, P. N., Carvalho, A. H. S., Nobre, V. N., \& Guimarães, A. P. (2020). Atuação da enfermagem no cenário da pandemia COVID-19. Revista Nursing, 23 (269): 4765-47-68

Sampaio, F., Sequeira, C., \& Teixeira, L. (2020). Nurses' Mental Health During the Covid-19 Outbreak. Journal of Occupational and Environmental Medicine: 10, 783-787. 10.1097/JOM.0000000000001987 
Research, Society and Development, v. 10, n. 15, e318101522741, 2021

(CC BY 4.0) | ISSN 2525-3409 | DOI: http://dx.doi.org/10.33448/rsd-v10i15.22741

Santos, T. B. S., Andrade, L. R., Vieira, S. L., Duarte, J. A., Martins, J. S., Rosado, L. B., \& Pinto, I. C. M. (2021). Contingência hospitalar no enfrentamento da COVID-19 no Brasil: problemas e alternativas governamentais. Ciência \& Saúde Coletiva, 26 (4): 1407-1418

Souza, N. V. D. O., Carvalho, E. C., Soares, S. S. S., Varella, T. C. M. M., Pereira, S. E. M., \& Andrade, K. B. S. (2021). Trabalho de enfermagem na pandemia da Covid-19 e repercussões para a saúde mental dos trabalhadores. Rev Gaúcha Enferm, 42(esp):e20200225. https://doi.org/10.1590/1983-1447.2021.20200225

Tobase, L., Cardoso, S. H., Rodrigues, R. T. F., \& Peres, H. H. C. (2021). Empathic listening: welcoming strategy for nursing Professional in coping with with the coronavirus pandemic. Revista Brasileira de Enfermagem, 74 e20200721. https://doi.org/10.1590/0034-7167-2020-0721

Werneck, G. L., \& Carvalho, M. S. (2020). A pandemia de COVID-19 no Brasil: crônica de uma crise sanitária anunciada. Cadernos de Saúde Pública, 36 (5): e00068820 